\title{
Die EU als regionaler und globaler Akteur
}

\author{
Stephan Keukeleire und Kolja Raube
}

\section{Inhalt}

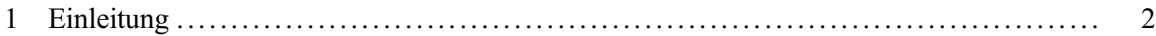

2 Selbstverständnis und Handlungsradius der EU als internationaler Akteur .............. 3

3 Die Herausforderung von Diversität und Differenz in der EU ..................... 4

4 Die Herausforderung von Kohärenz, Effizienz und Politiknexus .................... 6

5 Herausforderungen der internen und externen Legitimität ........................ 7

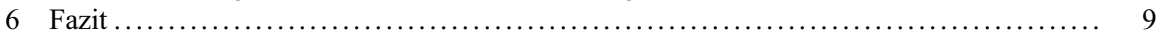

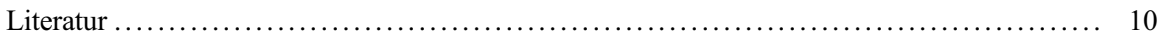

\section{Zusammenfassung}

Die Europäische Union tritt auf regionaler sowie globaler Ebene als internationaler Akteur auf. Die Europäische Union (EU) ist ein internationaler Akteur mit weltweitem Aktionsradius, der jedoch im Vergleich zu staatlichen Akteuren über eine Reihe von besonderen Eigenschaften verfügt. Diese Besonderheiten wirken sich auf die Qualität und Fähigkeiten der EU als internationaler Akteur aus. Zu den spezifischen Herausforderungen, vor denen die EU steht, zählen die Diversität und Differenz ihrer Institutionen und Mitgliedstaaten, Kohärenz und Effizienz ihres auswärtigen Handelns sowie Fragen der internen und externen Legitimität.

Wir möchten Franziska Petri für die wissenschaftliche Mitarbeit und Übersetzung des Artikels aus dem Englischen danken.

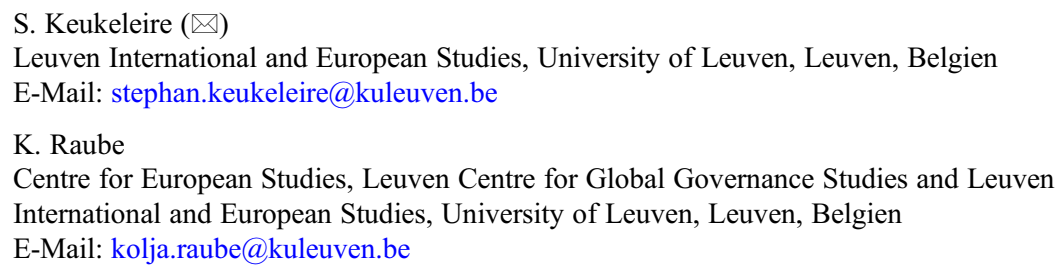


Ihre Qualitäten und Fähigkeiten als internationaler Akteur sind auf der einen Seite unter dem Gesichtspunkt ihrer Besonderheiten als Staatenverbund und Integrationsgemeinschaft $\mathrm{zu}$ verstehen. Auf der anderen Seite stellt sich die Frage, ob und wie die EU, hinsichtlich einer den europäischen und westlichen Werten und einer liberalen internationalen Politik zunehmend kritisch gegenüberstehenden Welt, einen Paradigmenwechsel in der Praxis des auswärtigen Handelns vollziehen muss.

\section{Schlüsselwörter}

Europäische Union · Globaler Akteur · Diplomatie · Internationale Beziehungen · Regional Governance

\section{$1 \quad$ Einleitung}

Das auswärtige Handeln der EU ist von der Segmentierung verschiedener Politikfelder geprägt (Damro et al. 2018), die weit über die Aufteilung in Gemeinsame Außen- und Sicherheitspolitik (GASP) und Gemeinsame Sicherheits- und Verteidigungspolitik (GSVP) hinausreicht. Mit anderen Worten, es reicht nicht mehr, das auswärtige Handeln durch die Politik im Bereich der intergouvernementalen GASP/ GSVP zu fassen, sondern es müssen auch andere (supranational organisierte) interne und externe Politikbereiche - u. a. Handels-, Entwicklungs-, Migrations-, Umweltpolitik - mit einbezogen werden. In der Tat umfasst die regionale und globale Akteursfähigkeit der EU alle Bereiche der Außenpolitik (Keukeleire und Delreux 2014; Hill et al. 2017): Diplomatie (Smith et al. 2016), Sicherheit und Verteidigung (Howorth 2014), Handel (Gstöhl und De Bièvre 2017; Khorona und Garcia 2018), Entwicklungszusammenarbeit (Orbie und Carbone 2016; Szent-Iványi und Lightfoot 2015), humanitäre Hilfe und spezifische interne Politiken mit externer Dimension, wie beispielsweise Umwelt-, Energie- oder Migrationspolitik (Damro et al. 2018; Knodt et al. 2017; Schunz 2014).

Dabei wird die EU nicht nur vor die schwierige Aufgabe gestellt, mitgliedstaatliche Interessen durch den Europäischen Auswärtigen Diensts (EAD) zu koordinieren, sondern auch die jeweiligen Politikbereiche kohärent zusammenzuführen und effizient umzusetzen sowie in verschiedenen bilateralen wie multilateralen Kontexten zu implementieren. Dass die EU hierbei zunehmend von außen für ihre westlichen Interessen und Normen kritisiert wird, stellt die EU vor ein zusätzliches Problem. Die EU sieht sich daher zunehmend aufgefordert, ihr Handeln im Äußeren zunehmend strategisch zu „unterfüttern“, so dass mitgliedstaatliche Interessen, Instrumente verschiedener Politikbereiche sowie die Anforderungen in einer sich stetig wandeln Welt zusammengedacht werden. 


\section{Selbstverständnis und Handlungsradius der EU als internationaler Akteur}

Das auswärtige Handeln der Europäischen Union (EU) umfasst jegliches über ihre Grenzen hinausgehende Tätigwerden. Die EU verfügt als außenpolitischer Akteur über ein weites Netzwerk an internationalen Beziehungen und ist auf vielen Feldern sowie auf einer Vielzahl an Ebenen der Außenbeziehungen tätig (Fröhlich 2014; Hill et al. 2017; Keukeleire und Delreux 2014; Müller-Brandeck-Bocquet und Rüger 2011; Peters und Wagner 2011): der globalen Ebene, inter- oder intra-regionalen Ebenen, auf multilateraler Ebene im Rahmen internationaler Organisationen sowie in bilateralen Handlungskontexten gegenüber anderen Regionen oder Staaten. Zu diesem Zweck hat die Europäische Union ein spezifisches ,post-westfälisches“ diplomatisches System herausgebildet (Spence und Bátora 2015), das aus über 130 EU-Delegationen weltweit und dem EAD als diplomatischem Hauptquartier in Brüssel besteht und an deren Spitze die Hohe Vertreterin der Union für Außenund Sicherheitspolitik und Vizepräsidentin der Europäischen Kommission steht (Austermann 2014; Kruse 2014; Müller-Brandeck-Bocquet und Rüger 2011; Müller-Brandeck-Bocquet und Rüger 2015). Zwar wird die Diplomatie der EU idealerweise von einer strategischen globalen Vision geleitet und umfasst Aktionen, welche auf eine strukturelle Veränderung globaler Lebensbedingungen abzielen (Smith et al. 2016), doch wird das europäische diplomatische Handeln gleichzeitig stark durch eine wachsende Segmentierung verschiedener Politikfelder geprägt (Damro et al. 2018), die weit über die Aufteilung in Gemeinsame Außen- und Sicherheitspolitik (GASP) und Gemeinsame Sicherheits- und Verteidigungspolitik (GSVP) hinausreicht. In der 2016 von der Hohen Vertreterin Federica Mogherini präsentierten „Globalen Strategie für die Außen- und Sicherheitspolitik der EU“ (oder „Globale Strategie der EU") folgt die EU diesem holistischen Ansatz einer einheitlichen Vision, die alle relevanten Politikfelder umfasst. Das Dokument verfolgt das ambitionierte Ziel, das auswärtige Handeln der Europäischen Union in einer „schwierige[n], zunehmend vernetzte[n], konfliktreiche[n] und komplexe[n] Welt" anzuleiten (EU 2016, S. 5; siehe auch EU 2015; Reiterer 2017; Tocci 2017; Winn und Gänzle 2017).

Damit deckt die Europäische Union als außenpolitischer Akteur zum einen die traditionelle ,relationale“ Außenpolitik ab, welche darauf abzielt, die Beziehungen zu sowie zwischen anderen Akteuren zu gestalten und zu beeinflussen. Zum anderen umfasst das außenpolitische Handeln der EU, aber auch die ,strukturelle“ Außenpolitik, welche auf die Gestaltung langfristiger Strukturen und ,rules of the game“ der internationalen Beziehungen abzielt und sich in Normdiffusionsprozessen als „external governance“ und insbesondere gegenüber Staaten in der europäischen Nachbarschaft widerspiegelt (Börzel und Risse 2012; Göler und Stratenschulte 2018; Keukeleire und Delreux 2014; Lavenex und Schimmelfennig 2009, 2010; Smith et al. 2016).

Als regionaler Akteur ist die Europäische Union vor allem in ihrer unmittelbaren Nachbarschaft aktiv: So haben beispielsweise die Staaten des Westbalkans mit Blick auf laufende EU-Erweiterungsverhandlungen sowie durch die bestehende Notwendigkeit, 
die zuvor kriegsgeprägte Region zu stabilisieren, eine besondere Aufmerksamkeit von Seiten der EU erhalten (Keil und Arkan 2015). Zudem stellt die Europäische Nachbarschaftspolitik (ENP) gegenüber Staaten ohne Aussicht auf EU-Mitgliedschaft das wichtigste Instrument ihrer regionalen Zusammenarbeit dar (Lippert 2017; Müller-Graff 2017). Die ENP richtet sich an Staaten Osteuropas und an die südliche Nachbarstaaten jenseits des Mittelmeers; beide Regionen werden zudem durch die Östliche Partnerschaft und die Union für das Mittelmeer unterstützt (Gstöhl und Schunz 2017; Lannon und Gstöhl 2015; Schumacher et al. 2018). Über die unmittelbare Nachbarschaft hinaus zeichnet sich die EU als regionaler Akteur außerdem durch interregionale Zusammenarbeit aus, insbesondere mit den AKPStaaten durch das Cotonou-Abkommen sowie die Kooperation mit anderen regionalen Organisationen wie ASEAN und Mercosur (Telò 2014).

Als globaler Akteur handelt die Europäische Union in einer Vielzahl internationaler Organisationen; sie positioniert sich selbst als Unterstützer des effektiven Multilateralismus (Bouchard et al. 2013; Panke 2014), hat jedoch gleichzeitig Schwierigkeiten, sich an die sich verändernde multilaterale Ordnung und sich ändernde Machtgleichgewichte anzupassen (Barbé et al. 2016; Drieskens und van Schaik 2014; Jorgensen und Laatikainen 2013). In diesem Zusammenhang hält die Debatte darüber an, welche Art von Macht die EU in ihrem Wesen sowie in ihrem Handeln in der Welt darstellt. Mit Blick auf die offizielle Rhetorik der europäischen Institutionen finden sich vielfach Referenzen auf das „Normative Power Europe“Konzept, welches lange Zeit einen großen Einfluss auf die Konzeptualisierung der europäischen Außenpolitik hatte (Forsberg 2011; Manners 2002; kritisch: Diez 2014). Dieser Perspektive zufolge handelt die EU im Äußeren nach ebenjenen Prinzipien, die sie im Inneren konstituieren: eben als ein normativ begründeter Akteur, dessen Handlungen an den Prinzipien der Rechtsstaatlichkeit und der Förderung der Menschenrechte ausgerichtet sind. Diese Sichtweise wurde in einer bis heute anhaltenden Debatte infrage gestellt, nicht zuletzt deshalb, weil der Einfluss der Mitgliedstaaten und ihre Interessen (Hyde-Price 2006) sowie der politische Wettbewerb in den Mitgliedstaaten und dessen Politisierung (Wagner 2017) im europäischen Außenhandeln unterrepräsentiert sind. Darüber hinaus hat das normative Image der EU auch im Zuge der internen Migrations- und Rechtsstaatlichkeitskrise (u. a. in Ungarn und Polen) Risse erhalten. Gleichwohl bleibt die EU in vielerlei Hinsicht ein mächtiger Akteur, insbesondere als Wirtschafts-, Handelsund Regulierungsmacht. (Damro 2015).

\section{Die Herausforderung von Diversität und Differenz in der EU}

Trotz der Versuche als einheitlicher Akteur zu handeln ist die „logic of diversity“ (Logik der Vielfalt) inhärent im auswärtigen Handeln der Europäischen Union (Hill 1998). Christopher Hill beschreibt dies so: „In that perpetual contest between homogeneity and difference, between lumping and splitting, which comes as close as any other to defining politics, European foreign policy is stuck with a full measure 
of „difference.“““ (Hill 1998, S. 36). Diese Logik der Differenz ist angesichts der Unterschiede der politischen Ziele und Interessen zwischen den Mitgliedstaaten und zwischen den europäischen Institutionen nicht überraschend.

So bestehen offensichtlich vertikale Differenzen zwischen Mitgliedstaaten und der EU in Bezug auf außenpolitische Interessen, nationale strategische Kulturen, Identitäten, Geschichte und Geografie - Faktoren, die zusammengenommen die Unterschiede in nationalen Außenpolitiken erklären können (Hadfield et al. 2017). Als Beispiele dienen die unterschiedliche Positionierung der EU-Mitgliedstaaten gegenüber internationalen Partnern, seien es die USA und die transatlantischen Beziehungen, China oder Russland. Diese Differenzen haben einen erheblichen Einfluss auf die EU als regionaler und globaler Akteur, da die Mitgliedstaaten eine zentrale Rolle innerhalb der Außenpolitik der EU spielen: Dies trifft für Entscheidungsprozesse innerhalb des Europäischen Rates oder des Ministerrates ebenso zu wie für Aktionen im Bereich der systematischen Zusammenarbeit zwischen Mitgliedstaaten, welche explizit in den europäischen Verträgen vorgesehen sind und einen wesentlichen Teil der EU-Diplomatie ausmachen (Bicchi und Maurer 2018; Keukeleire und Delreux 2014).

Zudem bestehen horizontale Differenzen zwischen den europäischen Institutionen im europäischen auswärtigen Handeln (Vanhoonacker und Pomorska 2017). So beeinflussen innerhalb des institutionellen Systems der EU die unterschiedlichen Kompetenzen zwischen den verschiedenen außenpolitischen Akteuren auf unterschiedlichen Politikfeldern deutlich die regionale und globale Handlungsfähigkeit der EU: Beinahe alle Bereiche des auswärtigen Handelns der EU fallen unter verschiedene Kompetenzbegrenzungen und sehen unterschiedliche Entscheidungsprozesse vor (Thym 2009). Dies führt in der Konsequenz dazu, dass eine Vielzahl von Akteuren in ungleichem Maße an der auswärtigen Politik der EU mitwirkt. Hinzu kommen jüngste Entwicklungen wie der Aufbau des Europäischen Auswärtigen Diensts (EAD) als „de novo body“ (Bickerton et al. 2015, S. 705) oder auch „interstitial organization“ (Bátora 2013; Duke 2017), ebenso wie der wachsende Einfluss des Europäischen Parlaments auf die Außenpolitiken der EU (Raube 2012; Rosén und Raube 2018), welche das Ausmaß an Differenzierung zwischen intergouvernementalen und supranationalen Akteuren noch weiter verstärken.

Neben diesen Dimensionen der internen Differenzen gilt es darüber hinaus externe Differenzen im Bereich der internationalen Beziehungen der EU (Keukeleire und Lecocq 2018). Diese externen Differenzen sind das Ergebnis einer zunehmenden Kritik an der bestehende internationalen Ordnung internationaler Beziehungen, die von westlichen Akteuren gegründet und nun u. a. von der EU unterstützt wird. Diese Kritik äußert sich nicht zuletzt in der Verfechtung alternativer Normen und Interessen verschiedener nicht-westlicher Akteure auf der Bühne internationaler Politik. Die Globale Strategie der EU reagiert auf diese Herausforderung (EU 2016, S. 3), indem sie unterstreicht, dass die EU neue „pragmatische“ Wege suchen muss, um auf lokale und internationale Entwicklungen angemessene Antworten zu finden. In Zeiten wachsender Interdependenz, und damit verbundenen unscharfen inneren und äußeren Grenzen, wird die Notwendigkeit für Problemlösungsprozesse jenseits des Nationalstaates erschwert durch die Berücksichtigung 
ebensolcher Unterschiede. Das auswärtige Handeln der EU ist somit mit der Frage konfrontiert, wie diese zwischen der EU und staatlichen Akteuren, internationalen Organisationen, transnationalen Akteuren und Zivilgesellschaft überbrückt werden können.

\section{Die Herausforderung von Kohärenz, Effizienz und Politiknexus}

Kohärenz ist von Natur aus eine Herausforderung für die Europäische Union, da ihre auswärtigen Politiken - z. B. die Außenhandelspolitik und die GASP - formal getrennt und zugleich miteinander verbunden sind. Gleichermaßen hängt die Effizienz der EU - im Sinne externer Handlungsfähigkeit - von ihrer Kohärenz ab. Wie Marangoni und Raube (2014) gezeigt haben, stellt so auch der Vertrag von Lissabon Kohärenz und Effizienz direkt nebeneinander:

\footnotetext{
„Die Union verfügt über einen institutionellen Rahmen, der zum Zweck hat, ihren Werten Geltung zu verschaffen, ihre Ziele zu verfolgen, ihre Interessen, denen ihrer Bürgerinnen und Bürger und denen der Mitgliedstaaten zu dienen sowie die Kohärenz, Effizienz und Kontinuität ihrer Maßnahmen sicherzustellen.“ (Art. 13 Abs. 1 EUV, Hervorhebung der Autoren)
}

Interessanterweise betonte bereits die Europäische Sicherheitsstrategie (ESS) von 2003 die Notwendigkeit einer größeren Kohärenz der EU-Instrumente und wiederholte den Aufruf für mehr Kohärenz, um den Einfluss der EU auf der internationalen Bühne zu stärken: „Wenn wir aber einen unserem Potenzial entsprechenden Beitrag leisten wollen, dann müssen wir noch aktiver, kohärenter und handlungsfähiger sein“ (EU 2003, S. 39). Die gleiche Botschaft drückte auch der Bericht zur Umsetzung der Europäischen Sicherheitsstrategie (2008) aus, in dem die Innovationen des Vertrags von Lissabon zur Verbesserung der kohärenten Nutzung der EU-Instrumente begrüßt wurden (EU 2008, S. 2).

Dementsprechend ist die EU gefordert, die Kohärenz ihrer Instrumente und Politiken entlang der vertraglichen Vorgabe zu verbessern, um ihre außenpolitischen Ziele erreichen zu können. Dabei lassen sich verschiedene Dimensionen von Kohärenz unterscheiden: Erstens muss die EU im Sinne der horizontalen Kohärenz auf größtmögliche Synergien der in verschiedenen Politikfeldern angewandten Instrumente abzielen und dabei Widersprüche verschiedener politikfeldspezifischer Ziele vermeiden (z. B. im Hinblick auf unterschiedliche Interessen in der Handels-, Sicherheits- und Entwicklungspolitk). Zweitens sollte die EU gemäß der institutionellen Kohärenz dafür sorgen, dass die verschiedenen Akteure innerhalb des EU-Institutionengefüges einheitliche Politiken formulieren und so Widersprüche zwischen den Positionen und Instrumenten verschiedener EU-Institutionen verhindern. Drittens sollten im Sinne der vertikalen Kohärenz die EU-Mitgliedstaaten und EU-Institutionen ihr jeweiliges außenpolitisches Vorgehen aufeinander abstimmen und gegenläufige Außenpolitiken auf nationalstaatlicher und EU-Ebene vermeiden. 
Schließlich muss die EU selbst in ihrem außenpolitischen Handeln externe Kohärenz gewährleisten, das heißt, ein vermeintlich widersprüchliches Handeln gegenüber Drittakteuren über Zeit oder gegenüber verschiedenen Akteuren vermeiden (Keukeleire und Raube 2013). Die Probleme der EU, beispielsweise auf Krisen und „Revolten“ in der arabischen Welt sowie der Ukraine angemessene Antworten zu finden, unterstreichen, wie schwer sich die EU tut, kohärent, effizient und kontinuierlich zu agieren, wenn Turbulenzen und Krisen die internatioanle Poitik bestimmen und Akteure internationale Normen unterlaufen oder anfechten. (Börzel et al. 2015; Horst et al. 2013; Schumacher et al. 2018). Andererseits war die EU im Falle des Nuklearabkommens mit Iran sehr wohl in der Lage, die Kohärenz, Effizienz und Kontinuität ihrer Politik zu garantieren, auch trotz der vielschichtigen Involvierung ihrer Mitgliedstaaten (insbesondere) in den fortlaufenden Verhandlungen (Adebahr 2017). Die EU war in der Tat ein effektiver diplomtischer Akteur in den Verhandlungen mit Iran, insbesondere durch die „EU3“ (Deutschland, Frankreich und Grossbritannien) auf der einen Seite sowie die Hohe Vertreterin der GSVP/Vizepräsidentin der Komission auf der anderen, die die anderen Mitgliedstaaten und die Kommission vertrat. Die Verhandlungen bewiesen einmal mehr, welch besonderen Akteur die EU in den internationalen Beziehungen darstellt, der auf der einen Seite die EU-Institutionen und auf der anderen Seite die nach wie vor einflussreichen Mitgliedstaaaten umfasst.

Inbesondere mit Blick auf institutionelle und horizontale Kohärenz ergeben sich somit vermeintliche Politikfeldverknüpfungen und -synergien, die unter anderem in den Politikfeldern Handel-Entwicklung, Sicherheit-Entwicklung oder Menschenrechte/Rechtsstaatlichkeit-Handel bereits vielfach identifiziert worden sind (Carbone und Orbie 2014; Keukeleire und Raube 2013). Gleichermaßen zeigen auch eine Reihe offizieller EU-Dokumente einen Nexus zwischen verschiedenen Bereichen der EU-Außenpolitik wie beispielsweise ,, Der Europäischer Konsens für Entwicklungspolitik“ (2006, 2017), das Strategiepapier der Kommission „Handel für alle“ (2015), die „Globale Strategie der EU“ (2016) und das jüngste Reflexionspapier der Kommission „Die Globalisierung meistern“ (2017).

\section{$5 \quad$ Herausforderungen der internen und externen Legitimität}

Mit der „Globalen Strategie für die Außen- und Sicherheitspolitik der EU“ hat die EU Grundsätze für das auswärtige Handeln der Europäischen Union in einer „schwierige[n], zunehmend vernetzte[n], konfliktreiche[n] und komplexe[n] Welt" formuliert (EU 2016, S. 5). Sie folgt der Einschätzung, dass nicht nur die Welt, sondern vor allem auch die EU und ihre Außenpolitik immer umstrittener (,contested"), also von innen wie von außen angefochten, wird. Vor diesem Hintergrund zeigt sich die Notwendigkeit für die EU, eine größere Legitimität und Glaubwürdigkeit ihres globalen Handelns zu erzielen; die EU muss das Vertrauen in ihr Handeln sowohl innerhalb der EU (interne Legitimität) als auch außerhalb der EU (externe Legitimität) wiederherstellen (Raube und Tonra 2018). 
Mit Blick auf die interne Legitimität sieht sich die EU mit einem inneren parlamentarischen Legitimitätsdefizit konfrontiert (Peters et al. 2014; Wagner 2010), weil die parlamentarische Kontrolle der verschiedenen Dimensionen des auswärtigen Handelns sowohl auf EU-Ebene als auch innerhalb der unterschiedlichen politischen Systeme der 28 EU-Mitgliedstaaten sehr ungleich ist (Raube und Wouters 2017). Gleichwohl haben in letzter Zeit nationale und subnationale Parlamente begonnen, eine größere Rolle für sich im Bereich der Handelspolitik zu fordern - obwohl diese unter die exklusiven Kompetenzen der EU fällt (RoedererRynning und Kallestrup 2017). Was das intergouvernemental geprägte Politikfeld der GASP/GSVP angeht - jenes Politikfeld, in dem die Rolle des Europäischen Parlaments oft nur als ,symbolisch“ bezeichnet wird (Ripoll Servent 2018) -, lässt sich ein wachsender Einfluss des Parlaments beobachten, insbesondere durch den Rückgriff auf Instrumente der Haushaltskontrolle (Rosén und Raube 2018; Raube 2012). Auch haben neu entstehende Formate der interparlamentarischen Kooperation, beispielsweise die Interparlamentarischen Konferenzen für die GASP und GSVP (Konrath und Liebich 2014), zu einem wachsenden parlamentarischen Informationsnetzwerk im Bereich der GASP/GSVP-Entscheidungsprozesse beigetragen.

Richtet man den Blick auf die Frage der externen Legitimität, so vollzieht sich die Außenpolitik der EU keineswegs in einem Vakuum, sondern in einem größeren globalen Kontext. Die Globale Strategie der EU beschreibt dies wie folgt: „Unsere Außen- und Sicherheitspolitik muss globalen Druck und lokale Dynamik meistern; sie muss mit Supermächten und zunehmend zerrütteten Identitäten umgehen können.“ (EU 2016, S. 3). Die Herausforderung der externen Legitimität nimmt somit verschiedene Formen an: Erstens operiert die EU im Kontext einer schwindenden Legitimität der bestehenden internationalen Ordnung, die als solche in der Nachkriegszeit von den westlichen Mächten gestaltet wurde und nunmehr von der EU gefördert wird. Dieses Legitimitätsproblem manifestiert sich in der veränderten Wahrnehmung der EU durch ihre internationalen Partner sowieden Aufstieg neuer multilateraler Bündnisse der aufkommenden Mächte des globalen Südens (Keukeleire und De Bruyn 2017; Knodt et al. 2017). Zweitens sieht sich die EU als regionaler und globaler Akteur zunehmend mit anderen mächtigen außenpolitischen Akteuren mit beachtlicher externer Legitimität (u. a. Russland, USA, China oder Saudia Arabien) konfrontiert. Drittens ist das Bild von einem internationalen System, das vorwiegend aus souveränen Nationalstaaten besteht, mittlerweile auch in der Politik umstritten: So betonte der strategische Bericht zur Lage der EU-Außenpolitik der Hohen Vertreterin von 2015 die besondere Rolle staatlicher, nicht staatlicher, zwischenstaatlicher und transnationaler Akteure, welche zusammengenommen eine ,world of overlapping webs“ konstituierten, was zu einer veränderten Bedeutung von Grenzen führe (EU 2015, S. 10, S. 4). Dies bedeutet auch, dass die EU in ihrer Außenpolitik verschiedenartige lokale Herrschaftsstrukturen miteinbeziehen sollte, da diese oft eine besonders starke Legitimität innerhalb ihrer Gesellschaften besitzen, wie beispielsweise religiöse Gruppierungen oder ethnisch begründete politische Strukturen im Nahen Osten und Afrika (Keukeleire und Lecocq 2018).

Diese Erkenntnis führt zu einer weiteren zentralen Herausforderung für die politische Praxis und die wissenschaftliche Analyse: zu dem Versuch, ein besseres 
Verständnis für eine nicht nur zunehmend vernetzte, komplexe und umstrittene, sondern darüber hinaus zunehmend nicht-europäische und nicht-westliche Welt zu erlangen (Fisher Onar und Nicolaïdis 2013). Dies impliziert, dass unsere Wissensgemeinschaft über traditionelle westliche Paradigmen hinaus blicken muss, um sowohl europapolitische Praktiker als auch Wissenschaftler zu einem besseren Verständnis der „Welt der vorhersehbaren Unvorhersehbarkeit“"zu führen sowie sich „die Ausrüstung zu[zu]legen, die uns ein schnelleres und flexibleres Reagieren auf künftige Ungewissheiten ermöglicht" und dadurch die „Wissensbasis, die unserem auswärtigen Handeln zugrunde liegt“, sowie ein „eingehenderes Lagebewusstsein“ zu stärken (EU 2016, S. 40-41).

In diesen Zusammenhang fügt sich der Aufruf des „Decentring“ für die Analyse und Praxis der europäischen Außenpolitik ein, der auf das Überwinden von Euround Westzentrismus abzielt (Fisher Onar und Nicolaïdis 2013; Keukeleire und Lecocq 2018). „Decentring“ bedeutet dabei, dass EU-Außenpolitik aus der Perspektive und innerhalb des Kontexts der Region, der Staaten oder Gesellschaften, die Gegenstand der EU-Außenpolitik sind, analysiert werden sollte - und dies nicht allein aus altruistischen Gründen, sondern um die Relevanz, Effizienz und Legitimität der EU als regionaler und globaler Akteur zu stärken. Eine solche „decentred perspective" kann sich als besonders ergiebig gegenüber anderen Regionen erweisen, wie beispielsweise dem Mittelmeerraum oder aber im Fall spezifischer außenpolitischer Ziele wie Demokratieförderung oder Rechtsstaatlichkeit in Osteuropa und darüber hinaus (Morozov 2013). Der „Decentring“-Ansatz trägt in diesem Sinne dazu bei, zu erkennen, dass das auswärtige Handeln der EU oftmals in nichteuropäischen Staaten und Gesellschaften durch die Brille der kolonialen Vergangenheit wahrgenommen wird. Das bedeutet, dass während die EU oftmals von einer wertegebundener Außenpolitik ausgeht (gebunden an westliche Werte wie Demokratie, Rechtstaatlichkeit und Menschenrechte), die EU, von außen betrachtet, wegen ihrer kolonialen Vergangenheit oder Beziehungen zu autokratischen Regimen als heuchlerisch und wenig glaubhaft angesehen wird. So kann das Verständnis dieser „Echoes of Empire“ (Nicolaidis et al. 2015), die weiterhin in anderen Teilen der Welt nachhallen, dabei helfen, den fehlenden Widerhall europäischer außenpolitischer Ziele in Drittstaaten und Regionen zu verstehen.

\section{$6 \quad$ Fazit}

Das globale Handeln der Europäischen Union ebenso wie ihre globale und normative Vision ist im Verlauf der letzten Jahre im Bestreben, Unionsinteressen strategisch zu stärken, zunehmend differenzierter und anspruchsvoller geworden. In einer globalisierten Welt hat die wachsende Interdependenz die Bedeutung des auswärtigen Handelns der EU für europäische Bürger und EU-Mitgliedstaaten deutlich gemacht. Gleichwohl ist zu erkennen, dass verschiedene interne und externe Herausforderungen der EU abverlangen, ihre Positionen und ihr Handeln im Weltgeschehen zu überdenken. Während die EU einerseits Wege gefunden hat, ihr auswärtiges Handeln zu koordinieren und durch die Globale Strategie neu auszurichten, 
fordern andererseits interne und externe zentrifugale Kräfte die bestehende europäische Ordnung sowie die internationale Ordnung in ihrer Gesamtheit heraus. Diese Kräfte beeinflussen, wie die EU wahrgenommen wird und ob ihr als globaler Akteur Vertrauen entgegengebracht wird. Die zentrale Frage für die Zukunft des auswärtigen Handelns der Europäischen Union wird daher sein, wie sich die EU inmitten der gegenwärtigen globalen Veränderungen positionieren wird und ob EU-Politiker und -Diplomaten bereit sind ,out of the box“ zu denken, um diesen Herausforderungen angemessen zu begegnen.

\section{Literatur}

Adebahr, Cornelius. 2017. Europe and Iran. The nuclear deal and beyond. London: Routledge.

Austermann, Frauke. 2014. European Union delegations in EU foreign policy: A diplomatic service of different speeds. Basingstoke: Palgrave Macmillan.

Barbé, Esther, Oriol Costa, und Robert Kissack, Hrsg. 2016. EU policy responses to a shifting multilateral system. London: Palgrave Macmillan.

Bátora, Jozef. 2013. The ,Mitrailleuse Effect": The EEAS as an interstitial organization and the dynamics of innovation in diplomacy. JCMS: Journal of Common Market Studies 51(4): 598-613.

Bicchi, Federica, und Heidi Maurer. 2018. Introduction. The Hague Journal of Diplomacy 13(1): $1-19$.

Bickerton, Christopher J., Dermot Hodson, und Uwe Puetter. 2015. The new intergovernmentalism: European integration in the Post-Maastricht Era. JCMS: Journal of Common Market Studies 53(4): 703-722.

Börzel, Tanja A., und Thomas Risse. 2012. Special issue: From Europeanisation to diffusion. West European Politics 35(1): 1-19.

Börzel, Tanja A., Assem Dandashly, und Thomas Risse. 2015. Special issue: Responses to the „Arabellions": The EU in comparative perspective. Journal of European Integration 37(1): 2015.

Bouchard, Caroline, John Peterson, und Nathalie Tocci, Hrsg. 2013. Multilateralism in the 21st century: Europe's quest for effectiveness. London: Routledge.

Carbone, Maurizio, und Jan Orbie. 2014. The trade-development nexus in the European Union: Differentiation, coherence and norms. London: Routledge.

Damro, Chad. 2015. Market power Europe: Exploring a dynamic conceptual framework. Journal of European Public Policy 22(9): 1336-1354.

Damro, Chad, Sieglinde Gstöhl, und Simon Schunz, Hrsg. 2018. European Union's evolving external engagement: Towards new sectoral diplomacies? London/New York: Routledge Taylor \& Francis Group.

Diez, Thomas, Hrsg. 2014. A different kind of power? The EU's role in international politics. New York: International Debate Education Association.

Drieskens, Edith, und Louise G. van Schaik. 2014. The EU and effective multilateralism: Internal and external reform practices. Hoboken: Taylor and Francis.

Duke, Simon. 2017. Europe as a stronger global actor - Challenges and strategic responses. Basingstoke: Palgrave Macmillan.

Europäische Kommission. 2015. Handel für alle. Hin zu einer verantwortungsbewussten Handelsund Investitionspolitik. Europäische Kommission. http://trade.ec.europa.eu/doclib/docs/2015/ october/tradoc_153880.PDF. Zugegriffen am 10.02.2018.

Europäische Kommission. 2017. Reflexionspapier. Die Globalisierung meistern. Europäische Kommission. https://ec.europa.eu/commission/sites/beta-political/files/reflection-paper-globali sation_de.pdf. Zugegriffen am 10.02.2018. 
Europäische Union. 2003. Europäische Sicherheitsstrategie. Ein sicheres Europa in einer besseren Welt. Consilium. http://www.consilium.europa.eu/de/documents-publications/publications/euro pean-security-strategy-secure-europe-better-world/. Zugegriffen am 10.02.2018.

Europäische Union. 2006. „Der Europäische Konsens“ für die Entwicklung. EUR-Lex. http://eurlex.europa.eu/legal-content/DE/TXT/?uri=LEGISSUM:r12544. Zugegriffen am 10.02.2018.

Europäische Union. 2008. Bericht über die Umsetzung der Europäischen Sicherheitsstrategie. Sicherheit schaffen in einer Welt im Wandel. Consilium. http://www.consilium.europa.eu/ ueDocs/cms_Data/docs/pressdata/DE/reports/104634.pdf. Zugegriffen am 10.02.2018.

Europäische Union. 2015. Strategic Review. The European Union in a changing global environment. Europa.eu. https://europa.eu/globalstrategy/en/strategic-review-european-union-chan ging-global-environment. Zugegriffen am 10.02.2018.

Europäische Union. 2016. Gemeinsame Vision, gemeinsames Handeln: Ein stärkeres Europa. Eine Globale Strategie für die Außen- und Sicherheitspolitik der Europäischen Union. Europa.eu. http://europa.eu/globalstrategy/sites/globalstrategy/files/eugs_de_0.pdf. Zugegriffen am 10.02.2018.

Europäische Union. 2017. Der „Neue Europäische Konsens über die Entwicklungspolitik“. Consilium. http://www.consilium.europa.eu/de/press/press-releases/2017/06/07/joint-strategy-euro pean-consensus-development/. Zugegriffen am 10.02.2018.

Fisher Onar, Nora, und Kalypso Nicolaïdis. 2013. The decentring agenda: Europe as a post-colonial power. Cooperation and Conflict 48(2): 283-303.

Forsberg, Tuomas. 2011. Normative power Europe, once again: A conceptual analysis of an ideal type*. JCMS: Journal of Common Market Studies 49(6): 1183-1204.

Fröhlich, Stefan. 2014. Die Europäische Union als globaler Akteur: Eine Einführung, 2. Aufl. Wiesbaden: Springer VS.

Göler, Daniel, und Eckart J. Stratenschulte. 2018. Norm- und Regeltransfer in der europäischen Außenpolitik. Schriftenreihe des Arbeitskreises Europäische Integration e.V., Bd. 99. BadenBaden: Nomos.

Gstöhl, Sieglinde, und Dirk de Bièvre. 2017. The trade policy of the European Union. Oxford/London: Macmillan Education; Palgrave.

Gstöhl, Sieglinde, und Simon Schunz, Hrsg. 2017. Theorizing the European neighbourhood policy. London/New York: Routledge Taylor \& Francis Group.

Hadfield, Amelia, Ian Manners, und Richard G. Whitman, Hrsg. 2017. Foreign policies of EU member states: Continuity and Europeanisation. London/New York: Routledge Taylor \& Francis Group.

Hill, Christopher. 1998. Convergence, divergence and dialectics: National foreign policies and CFSP. In Paradoxes of European foreign policy, Hrsg. Jan Zielonka, 35-52. Den Haag: Kluwer Law International.

Hill, Christopher, Michael Smith, und Sophie Vanhoonacker, Hrsg. 2017. International relations and the European Union. Oxford: Oxford University Press.

Horst, Jakob, Annette Jünemann, und Delf Rothe, Hrsg. 2013. Euro-Mediterranean relations after the Arab Spring, persistence in times of change. Farnham: Ashgate.

Howorth, Jolyon. 2014. Security and defence policy in the European Union, 2. Aufl. Basingstoke: Palgrave Macmillan.

Hyde-Price, Adrian. 2006. ,Normative“ power Europe. A realist critique. Journal of European Public Policy 13(2): 217-234.

Jorgensen, Knud Erik, und Katie Laatikainen, Hrsg. 2013. Routledge handbook on the European Union in international institutions: Performance, policy, power. New York: Routledge.

Keil, Soeren, und Zeynep Arkan, Hrsg. 2015. The EU and member state building: European foreign policy in the Western Balkans. London/New York: Routledge.

Keukeleire, Stephan, und Tom De Bruyn. 2017. The European Union, the BRICs, and other Emerging powers: Towards a new world order. In International relations and the European Union, Hrsg. Christopher Hill, Michael Smith und Sophie Vanhoonacker, 418-444. Oxford: Oxford University Press. 
Keukeleire, Stephan, und Tom Delreux. 2014. The foreign policy of the European Union, 2. Aufl. Basingstoke: Palgrave Macmillan.

Keukeleire, Stephan, und Sharon Lecocq. 2018. Operationalizing the decentring agenda: Analysing European foreign policy in a non-European and post-Western World. Cooperation and Conflict 53(2): 277-295.

Keukeleire, Stephan, und Kolja Raube. 2013. The security-development nexus and securitisation in the EU's policies towards developing countries. Cambridge Review of International Relations 26(3): 556-572.

Khorona, Sangeeta, und Maria Garcia. 2018. Handbook on the EU and international trade. Cheltenham: Edward Elgar Publishing.

Knodt, Michèle, Natalia Chaban, und Louise Nielsen. 2017. Bilateral energy relations between the $E U$ and emerging powers. Baden-Baden: Nomos.

Konrath, Christoph, und David Liebich. 2014. Interparlamentarische Zusammenarbeit in der EU. Zeitschrift für Öffentliches Recht 69(3): 315-250.

Kruse, Franziska. 2014. Der Europäische Auswärtige Dienst zwischen intergouvernementaler Koordination und supranationaler Repräsentation. Berlin: Duncker \& Humblot.

Lannon, Erwoan, und Sieglinde Gstöhl. 2015. The European Union's broader neighbourhood: Challenges and opportunities for cooperation beyond the European neighbourhood policy. Abingdon: Routledge.

Lavenex, Sandra, und Frank Schimmelfennig. 2009. EU rules beyond EU borders: Theorizing external governance in European politics. Journal of European Public Policy 16(6): 791-812.

Lavenex, Sandra, und Frank Schimmelfennig, Hrsg. 2010. EU External Governance: Projecting EU rules beyond membership. London: Routledge.

Lippert, Barbara. 2017. Europäische Nachbarschaftspolitik. In Jahrbuch der Europäischen Integration 2017, Hrsg. Werner Weidenfeld und Wolfgang Wessels, 389-394. Baden-Baden: Nomos.

Manners, Ian. 2002. Normative power Europe: A contradiction in terms? Journal of Common Market Studies 40(2): 235-258.

Marangoni, Anne-Claire, und Kolja Raube. 2014. Virtue or vice? The coherence of the EU's external policies. Journal of European Integration 36(5): 473-489.

Morozov, Viatcheslav, Hrsg. 2013. Decentring the west: The idea of democracy and the struggle for hegemony. Surrey: Ashgate.

Müller-Brandeck-Bocquet, Gisela, und Carolin Rüger. 2011. The high representative for the EU foreign and security policy - Review and prospects. Baden-Baden: Nomos.

Müller-Brandeck-Bocquet, Gisela, und Carolin Rüger. 2015. Die Außenpolitik der EU. Berlin: De Gruyter Studium.

Müller-Graff, Peter-Christian, Hrsg. 2017. EU-Nachbarschaftspolitik - Nordafrika und Nahost. Schriftenreihe des Arbeitskreises Europäische Integration e.V., Bd. 102. Baden-Baden: Nomos.

Nicolaidis, Kalypso, Berny Sèbe, und Gabrielle Maas. 2015. Echoes of empire: Memory, identity and colonial legacies. New York/London: I.B. Tauris.

Orbie, Jan, und Maurizio Carbone. 2016. The Europeanisation of development policy. European Politics and Society 17(1): 1-11.

Panke, Diana. 2014. The European Union in the united nations. An effective external actor? Journal of European Public Policy 21(7): 1050-1066.

Peters, Dirk, und Wolfgang Wagner. 2011. Between military efficiency and democratic legitimacy: Mapping parliamentary war powers in contemporary democracies, 1989-2004. Parliamentary Affairs 64(1): 175-192.

Peters, Dirk, Wolfgang Wagner, und Cosima Glahn. 2014. Parliamentary control of CSDP: The case of the EU's fight against piracy off the Somali coast. European Security 23(4): 430-448.

Raube, Kolja. 2012. The European external action service and the European parliament. The Hague Journal of Diplomacy 7(1): 65-80.

Raube, Kolja, und Jan Wouters. 2017. The many facets of parliamentary involvement and interaction in EU external relations. In National parliaments after the Lisbon treaty and the Euro 
Crisis: Resilience or resignation, Hrsg. Davor Jancic, 281-298. Oxford: Oxford University Press.

Raube, Kolja, und Ben Tonra. 2018. Legitimacy in European Foreign and Security Policy (Special Issue). Global Affairs. (im Erscheinen).

Reiterer, Michael. 2017. Die Globale Strategie der Europäischen Union - den Visionen Taten folgen lassen. Integration 40(1): 11-30.

Ripoll Servent, Ariadna. 2018. The European parliament. Houndsmill: Palgrave Macmillan.

Roederer-Rynning, Christilla, und Marten Kallestrup. 2017. National parliaments and the new contentiousness of trade. Journal of European Integration 39(7): 811-825.

Rosén, Guri, und Kolja Raube. 2018. Influence beyond formal powers: Parliamentarization of European Union security policy. The British Journal of Politics and International Relations 20(1): 69-83.

Schumacher, Tobias, Andreas Marchetti, und Thomas Demmelhuber, Hrsg. 2018. The Routledge handbook on the European neighbourhood policy. Abingdon: Routledge.

Schunz, Simon. 2014. European Union foreign policy and the global climate regime. Brussels: Peter Lang.

Smith, Michael, Stephan Keukeleire, und Sophie Vanhoonacker, Hrsg. 2016. The diplomatic system of the European Union: Evolution, change and challenges. London: Routledge.

Spence, David, und Jozef Bátora, Hrsg. 2015. The European external action service: European diplomacy post-Westphalia. Basingstoke: Palgrave Macmillan.

Szent-Iványi, Balázs, und Simon Lightfoot. 2015. New Europe's new development aid. Abingdon: Routledge.

Telò, Mario, Hrsg. 2014. European Union and new regionalism: Competing regionalism and global governance in a post-hegemonic era. Aldershot: Ashgate.

Thym, Daniel. 2009. Foreign affairs. In Principles of European constitutional law, Hrsg. Armin von Bogdandy und Jürgen Bast, 309-343. Oxford: Hart Publishing.

Tocci, Nathalie. 2017. Framing the EU global strategy. A stronger Europe in a Fragile World. Cham: Springer-Palgrave Macmillan.

Vanhoonacker, Sophie, und Karolina Pomorska. 2017. The institutional framework. In International relations and the European Union, Hrsg. Christopher Hill, Michael Smith und Sophie Vanhoonacker, 97-122. Oxford: Oxford University Press.

Wagner, Wolfgang. 2010. Die demokratische Kontrolle internationalisierter Sicherheitspolitik. Baden-Baden: Nomos.

Wagner, Wolfgang. 2017. Liberal power Europe. Journal of Common Market Studies 55(6): $1398-1414$.

Winn, Neil, und Stefan Gänzle. 2017. Die Globale Strategie für die Außen- und Sicherheitspolitik der Europäischen Union - Zentralasien und der Südkaukasus: vom normativen Ansatz zum „prinzipiengeleiteten Pragmatismus“. Integration 40(4): 308-318. 